\title{
Letter to the Editor concerning "Comparison of combined anterior-posterior approach versus posterior-only approach in treating adolescent idiopathic scoliosis: a meta-analysis" by Chen Z, Rong L (2016) Eur Spine J;25(2):363-371
}

\author{
Adrian Gardner ${ }^{1}$
}

Received: 9 February 2016/Revised: 1 August 2016/Accepted: 1 August 2016/Published online: 17 August 2016

(C) Springer-Verlag Berlin Heidelberg 2016

\section{Dear Sir,}

I have read with interest the article titled 'Comparison of combined anterior-posterior approach versus posterior-only approach in treating adolescent idiopathic scoliosis: a meta-analysis' (Eur Spine J (2016) 25: 363-371) and would like to raise some concerns.

As stated in the article, there are only 10 studies that are either retrospective or non-randomised. This is a poor substrate to start this analysis. When examined further (Table 1) the surgical techniques employed differ and include anterior release (AR) and hybrid instrumentation versus posterior only pedicle screw constructs in at least two of the studies. Pedicle screws effect greater correction than hybrid systems so a comparison of AR and hybrid vs pedicle screws (PS) for the hypothesis here is flawed [1].

The sizes of curves in the AR group to posterior only (P) group are different in several of the studies. I appreciate this is why there has been an attempt to look at amount of correction rather than absolute values. Is it known whether the coronal Cobb will measure the same degree of correction in severe curves versus smaller curves given the greater degree of rotation in severe curves? Is it a fair comparison and a true measure of the amount of correction achieved? Additionally, the measurement of thoracic kyphosis (TK) has variation in technique [2] which may induce error in this assessment if not standardised across all studies. The measures of blood loss, length of stay, respiratory function and complications are greater in the AP vs

Adrian Gardner

adriangardnerd@googlemail.com

1 The Royal Orthopaedic NHS Foundation Trust, Bristol Road South, Northfield, Birmingham B31 2AP, UK
$\mathrm{P}$ group. This is what would be expected given the increased magnitude of surgery. These measures only become of importance if the primary outcome measure (correction) is equivalent or better for $\mathrm{P}$ versus $\mathrm{AP}$ correction.

A commonly stated aim of anterior surgery is 'to save a distal level'. This is important as this relates to long-term function [3]. It is unfortunate that it has not been possible to make a conclusion on this question in this analysis, as this remains an unanswered question for anterior surgery both the thoracic and thoracolumbar curves.

Therefore, I suggest that although the aims of this paper are laudable, the conclusions drawn are incomplete. Whilst a rigorous statistical analysis has been undertaken, I am concerned that the differences between the studies mean that this analysis was flawed even before it started. The only way to fully answer the question raised is prospective, randomised and matched comparison using identical techniques and implants apart from the AR.

Compliance with ethical standards

Conflict of interest The author of this letter confirms that there are no conflicts of interest.

\section{References}

1. Winter RB, Lonstein JE, Denis F (2007) How much correction is enough? Spine 32(24):2641-2643

2. Marks M, Stanford C, Newton P (2009) Which lateral radiographic positioning technique provides the most reliable and functional representation of a patient's sagittal balance? Spine 34(9):949-954

3. Fabricant PD, Admoni S-H, Green DW, Ipp LS, Widmann RF (2012) Return to athletic activity after posterior spinal fusion for adolescent idiopathic scoliosis: analysis of independent predictors. J Pediatr Orthop 32(3):259-265 